\title{
Biogenic Hydroxyapatite: A New Material for the Preservation and Restoration of the Built Environment
}

\author{
Ronald J Turner, Joanna C Renshaw, and Andrea Hamilton* \\ Department of Civil and Environmental Engineering, University of Strathclyde, Glasgow \\ E-mail: Andrea.Hamilton@strath.ac.uk
}

Keywords: Cement, Hydroxyapatite, Biomineralisation, Bioceramics, Medical Implant Materials, Heritage Conservation, Nuclear Waste Disposal, Environmental Remediation

\begin{abstract}
Ordinary Portland Cement (OPC) is by weight the world's most produced manmade material, and consequently is used in a variety of applications in environments ranging from buildings, to nuclear wasteforms, and within the human body. In this paper, we present for the first time the direct deposition of biogenic hydroxyapatite onto the surface of OPC, in a synergistic process which relies upon the unique properties of the cement substrate. The synthesised hydroxyapatite is very similar to that found in nature; having a crystallite size, iron and carbonate substitution, and a semi-crystalline structure which identify that this biogenic hydroxyapatite is akin to that found in natural bone and tooth enamel. Hydroxyapatites with such a structure are known to be mechanically stronger and more biocompatible than synthetic or biomimetic hydroxyapatites. The formation of this biogenic hydroxyapatite coating therefore has significance in a range of contexts. In medicine, hydroxyapatite coatings are linked to improved biocompatibility of ceramic implant materials. In the built environment, hydroxyapatite
\end{abstract}


coatings have been proposed for the consolidation and protection of sculptural materials such as marble and limestone, with biogenic hydroxyapatites having reduced solubility in comparison with synthetic apatites. Hydroxyapatites have also been established as effective for the adsorption and remediation of environmental contaminants such as radionuclides and metals. We further identify that in addition to providing a biofilm scaffold for nucleation, the metabolic activity of Pseudomonas fluorescens increases the $\mathrm{pH}$ of the growth medium to a suitable level for hydroxyapatite formation. The generated ammonia reacts with phosphate in the growth medium, producing ammonium phosphates, which are a precursor to the formation of hydroxyapatite under conditions of ambient temperature and pressure. Subsequently, this biogenic deposition process takes place in a simple reaction system under mild chemical conditions, which are cheap and easy to apply to fragile biological or architectural surfaces.

\section{Introduction}

Hydroxyapatite (HAP) is a calcium phosphate mineral with the general chemical formula $\mathrm{Ca}_{10}\left(\mathrm{PO}_{4}\right)_{6}(\mathrm{OH})_{2} \cdot{ }^{1} \mathrm{HAP}$ is an example of a 'bioceramic', ${ }^{2}$ a novel class of materials with potential applications ranging from bone implants, ${ }^{3}$ to drug delivery. ${ }^{4}$ Hydroxyapatite coatings have been used to reinforce bone cements, enhancing load-bearing capacity, ${ }^{5}$ and promoting proliferation and differentiation of human osteoblast-like cells. ${ }^{6,7}$ In addition, there is an emerging interest in the use of hydroxyapatite coatings for the consolidation and preservation of marble and limestone buildings and sculptures. ${ }^{8-12}$

Cement and concrete have been commonly used building materials throughout the 20th Century and there is significant interest in repairing concrete structures to reduce the amount of cement consumed worldwide. Cement is also used as a encapsulant for radioactive waste storage and the use of hydroxyapatites for the remediation of radionuclide ${ }^{13,14}$ and heavy metal $^{15}$ contaminants from the environment has been a subject of recent research interest.

The crystal structure of naturally-occuring HAP is too complex to be accurately mimicked 
by synthetic crystalline apatites, and the use of synthetic apatites has been observed to result in poor adhesion and low mechanical strength of dental treatments,${ }^{16}$ plus reduced radionuclide sorption capacity. ${ }^{13}$ Hydroxyapatite produced through the direct actions of a living organism - termed 'biogenic' hydroxyapatites - may offer a solution to these limitations, via intrinsic properties such as reduced solublity, and comparable particle size to those found in natural hydroxyapatites. ${ }^{16-18}$

Here we identify and describe a new method for deposition of a biogenic hydroxyapatite onto the surface of OPC; using the Gram negative bacterium Pseudomonas fluorescens. Unlike existing techniques, this method does not require the addition of an external calcium source and uses a simple bioreactor design with a common environmental microorganism. Importantly it is achieved using few reagents, requiring only Pseudomonas fluorescens, LB broth, and a phosphate $\mathrm{pH}$ buffer. Unlike synthetically grown $\mathrm{HAP}^{16,19}$ and biomimetic $\mathrm{HAPs},{ }^{20}$ this phase is easily formed at room temperature and pressure, making it applicable to the building industry and relatively economical.

The location, crystal structure, chemistry and morphology of the hydroxyapatite produced on OPC are investigated using XRD, FT-IR, and SEM-EDX. We identify that this biogenic hydroxyapatite has similarity to natural hydroxyapatites; which is of relevance to a range of applications, particularly nuclear waste disposal, medical implant materials, and architectural preservation. We additionally identify and describe for the first time the bacterially mediated biochemical mechanism behind this deposition process, which relies upon the metabolic generation of ammonium phosphate minerals.

\section{Methods}

\subsection{OPC Sample Preparation}

OPC Powder 'Multicem 32,5R (CEM-II/A-LL)' (Hanson Heidelberg) was hydrated with $200 \mathrm{~g}$ water per $500 \mathrm{~g}$ OPC powder (water:cement ratio $=0.4$ ). The paste was mixed for 
15 minutes using a Rotary Mixer on a low speed setting. The paste was then poured into silicone moulds and stored at $100 \%$ relative humidity and $20^{\circ} \mathrm{C}$ for 24 hours. Samples were removed and cured in a saturated solution of $\mathrm{Ca}(\mathrm{OH})_{2}\left(1.5 \mathrm{gL}^{-1}\right)$ for 28 days at $20^{\circ} \mathrm{C}$. The cement coupons were stored in this solution at $20^{\circ} \mathrm{C}$ until required.

\subsection{Biofilm Growth on Cured OPC Coupons}

Growth medium was prepared from $20 \mathrm{gL}^{-1} \mathrm{LB}$ Broth (Sigma-Aldrich), 9.4gL-1 $\mathrm{KH}_{2} \mathrm{PO}_{4}$ (Sigma-Aldrich), 2.2gL-1 $\mathrm{K}_{2} \mathrm{HPO}_{4}$ (Fluka), dissolved in deionised water in a 2L Duran flask, The flask was heated on a magnetic stirrer plate at $70^{\circ} \mathrm{C}$ to dissolve all components. Then $100 \mathrm{ml}$ volumes were decanted into Erlenmeyer flasks which were sealed with non-absorbent cotton wool bungs (Cowens Ltd) and autoclaved. OPC samples were rinsed thoroughly in DI water, and aseptically transferred into thel Erlenmeyer flasks ( 1 coupon per flask). The inoculum of $P$. fluorescens strain SBW25 was grown overnight at ambient temperature with continual shaking, in $100 \mathrm{ml}$ LB broth to an OD600 $=0.1$. Test flasks were inoculated with 100uL of this overnight culture. Control flasks were not inoculated. All flasks were incubated at ambient temperature on a shaking tray, inside a fume hood for 20 days. Control and test samples were aseptically retrieved from the flasks after 20 days and rinsed thoroughly in DI water to remove any biofilm material, then vacuum packed to minimise cement carbonation prior to analysis. To determine if contaminating bacteria were present on the control samples, and verify that Pseudomonas was growing successfully on test samples, scrapings from the surface were taken, homogenised via sonication, inoculated onto Tryptone Soya Agar plates (Oxoid), and incubated for 4 days at $20^{\circ} \mathrm{C}$. No contamination was observed on the control samples, and Pseudomonas growth was apparent on all test samples.

\subsection{Examination of Ammonium Phosphate Mineral Formation}

Growth medium was prepared as described above, but with the addition of $15 \mathrm{gL}^{-1}$ agar (Sigma-Aldrich) prior to autoclaving. The medium was then aseptically dispensed into petri 
dishes, cooled, and stored overnight at room temperature. The following day, 3x plates were streak-inoculated with $P$. fluorescens culture overnight. 3x plates were kept as noninoculated controls. All plates were then incubated at $20^{\circ} \mathrm{C}$ for 12 days and $\mathrm{pH}$ was measured. After a 6 day incubation period, the plates were imaged and a fine opaque crystalline precipitate was observed within the bacterial colonies when viewed with an optical microscope (Nikon Eclipse LV100IVD) using a 10x objective lens and reflected light. There was no bacterial growth or precipitate observed on the control plates. After a 12 day incubation period large crystal precipitates were observed, associated with the bacterial colonies on the test plates. The crystals were imaged under a 10x objective lens, using cross-polarised light microscopy in incident mode. Still, no bacterial growth or crystal precipitates were observed on the control plates.

\subsection{SEM-EDS Analysis}

Polished thin cross-sections of the cement samples were made by resin impregnation followed by grinding and polishing the sample to a final thickness of $30 \mu \mathrm{m}$. For analysis sections were gold coated and imaged using an S-3700 SEM (Hitachi) fitted with an 80mm X-Max detector (Oxford Instruments) for EDS analysis. All SEM Images and EDS Maps were gathered at a $10 \mathrm{~mm}$ working distance and $15.0 \mathrm{kV}$ accelerating voltage.. SEM images without EDS analysis were collected using an SU6600 SEM (Hitachi) and all images were processed using ImageJ. ${ }^{21}$

\subsection{X-ray Diffraction (XRD) analysis}

XRD patterns were collected through the body of intact samples and smears from bacterial colonies grown on agar plates using an X-ray Diffractometer (Bruker D8 Advance). Patterns were collected in the 2 theta scan range $6-60^{\circ}$ using a step size of $0.02^{\circ}$, and a scan speed of 0.5 seconds/step. A Cu shutter tube generating X-rays with a wavelength of 1.5418 Angstrom $(0.15418 \mathrm{~nm})$ was used with a $0.6 \mathrm{~mm}$ X-ray slit. Samples were continually rotated during pattern collection. XRD data was processed using DiFFRAC EVA software (Bruker) and 
compared with mineral phases from the Inorganic Crystal Structure Database (ICSD). An estimate of hydroxyapatite crystallite size was calculated using Scherrer's equation, ${ }^{22}$ based on the full width at half maximum (FWHM) of the [002] reflection at $26^{\circ} 2$ theta.

\subsection{X-ray Fluorescence (XRF) Analysis}

All samples were ground into a fine powder prior to analysis. XRF analysis of OPC powder and fully cured hydrated OPC paste powders was carried out using a Bruker S4 Explorer instrument.

\section{$2.7 \quad$ FT-IR Analysis}

IR spectra were recorded using a Bruker Vertex 70 Fourier Transform Infrared Spectrometer. Representative portions of the sample surface were transferred on to the sample area of a single reflection diamond attenuated total reflectance (DATR) accessory, fitted with a KRS-5 substrate.

\subsection{Confocal Microscopy of Biofilms}

Biofilm-coated OPC samples were removed from the incubation flasks and rinsed in a $1 \mathrm{x}$ concentration phosphate buffered saline (PBS) solution. The samples were then transferred into a $50 \mathrm{ml}$ staining bottle containing $0.1 \% \mathrm{w} / \mathrm{v}$ acridine orange (Alfa-Aesar) in $1 \mathrm{x}$ PBS solution. The biofilms were stained for 5 minutes, removed from the solution, and then examined using a confocal microscope (Leica SP5 II). A laser excitation wavelength of 458nm was used to excite the fluorescent stain, and a photon multiplier tube with an emission collection wavelength of 475-550nm was used to collect the image data from the sample. Images was carried out using a 40x dry objective lenses and confocal maps were collected using 100 frames per optical section. 


\section{Results and discussion}

\subsection{Characterisation of Calcium Phosphates on OPC Samples}

OPC samples were incubated in phosphate-buffered LB broth (initial pH 6.2) with (test) and without (control) P. fluorescens inoculum for 20 days and then analysed using SEM-EDS, XRD and FT-IR.

Calcium phosphate mineralisation occurred on the surface of all OPC samples incubated in the medium, regardless of the presence of $P$. fluorescens. SEM-EDS analyses clearly demonstrate the presence of a calcium phosphate layer on the surface of the test sample, which was not observed on the untreated OPC control (Fig 1).

However, XRD (Fig 2A) and FT-IR (Fig 3) analysis showed that the phosphate layer deposited on the control sample was brushite.This could be expected, as the pH of the control solution was 7.4 , which is too low to precipitate hydroxyapatite. ${ }^{23}$ However, on samples incubated with Pseudomonas, biofilm growth was evident and associated with the formation of a hydroxyapatite layer on the OPC surface (Figs 1A, 2B, 3), forming below the biofilm. The location of the phosphate layer presents opportunities for generating hydroxyapatite coated cements, which could overcome the limited mechanical performance, such as low fracture toughness of synthetic calcium-phosphate based ceramics. ${ }^{24,25}$

The broad X-ray diffraction reflections in the XRD spectrum (Fig 2B) indicate that the bacterially deposited hydroxyapatite is a poorly crystalline material, particularly when compared to the sharp reflections from brushite (Fig 2A) observed on the control samples. Bio-

genic hydroxyapatite typically consists of poorly crystalline carbonated hydroxyapatite. ${ }^{26,27}$ In contrast, non-biologically generated calcium phosphate minerals typically present higher crystallinity. ${ }^{20}$ Further comparisons of our hydroxyapatite with a carbonate substituted hydroxyapatite (ICSD-289992) and a synthetic hydroxyapatite (ICSD-203027) identifies that the hydroxyapatite generated by Pseudomonas fluorescens appears most similar to the carbonated hydroxyapatite (Supplementary Information Fig 1, 2). 
Biological hydroxyapatites have been observed to incorporate different ions into their crystal structures. ${ }^{27}$ The FT-IR spectrum of the bacterially deposited hydroxyapatite (Fig 3B) displays peaks at 1456,1415 , and $874 \mathrm{~cm}^{-1}$. These peaks indicate the presence of carbonate ions $\left(\mathrm{CO}_{3}\right)^{-2}$, partially replacing $\left(\mathrm{PO}_{4}\right)^{-3}$ sites in the hydroxyapatite crystal structure. ${ }^{27}$ This creates an inherent disorder when compared with synthetic hydroxyapatites, which have higher crystallinity. ${ }^{20}$ Carbonate substitution of phosphate groups is a common characteristic of other biogenic hydroxyapatite crystals described in the literature. ${ }^{20}$ Additionally, the XRD pattern of the hydroxyapatite produced matches to that of 289992-ICSD - a calculated structure for a carbonate-substituted hydroxyapatite (Supplementary Fig 1). ${ }^{28}$ These observations indicate that this bacterial hydroxyapatite is comparable to natural hydroxyapatites found in bone and dental enamels, in terms of both ionic substitution and crystal structure.

The hydroxyapatite layer was observed to consist of a granular assembly of individual nanoscale particles, with size ranging from 30-100nm (Fig 3A). The observed morphology is consistent with nano-hydroxyapatite morphologies reported in the literature. ${ }^{16,29}$

Application of Scherrer's equation to the [002] reflection of the biogenic hydroxyapatite XRD patterns determined the crystallite size to be approximately 30nm (Fig 2B). This is similar to the particle size of natural hydroxyapatite found in teeth ${ }^{24}$ and corresponds well to literature values for biologically generated hydroxyapatites made using this same methodology; sizes typically ranging from $20-40 \mathrm{~nm} .{ }^{22,30}$ 

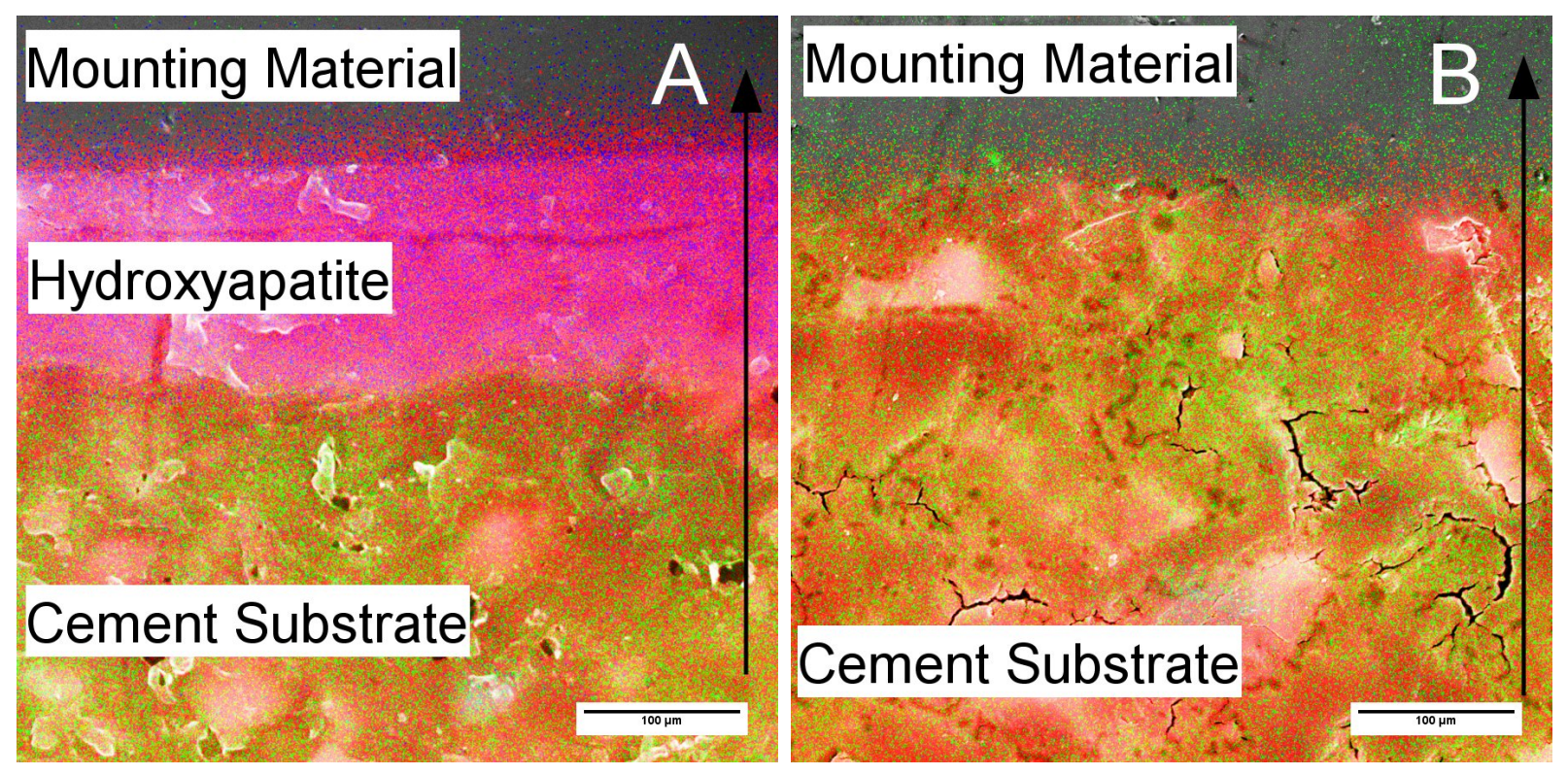

Figure 1: A) SEM-EDS map of the test OPC sample cross section. Image illustrates the interface at the top surface of the sample against the mounting material. The SEM-EDS map shows distribution of calcium (Red), silicon (Green) and phosphorous (Blue) through the sample cross-section. A calcium phosphate layer (Magenta) is clearly visible at the interface between the cement and the sample mounting material, representing the 'top' surface where the biofilm has been active. B) SEM-EDS Map of unmodified OPC sample cross-section cured for 28 days with no further modification. Image illustrates the interface at the top surface of the sample against the mounting material. The SEM-EDS map of calcium (Red) and silicon (Green) distribution in the sample is shown. No phosphorous was detected during EDS mapping of the control sample. The direction of the black arrows indicate the 'top' of the sample cross-section. 

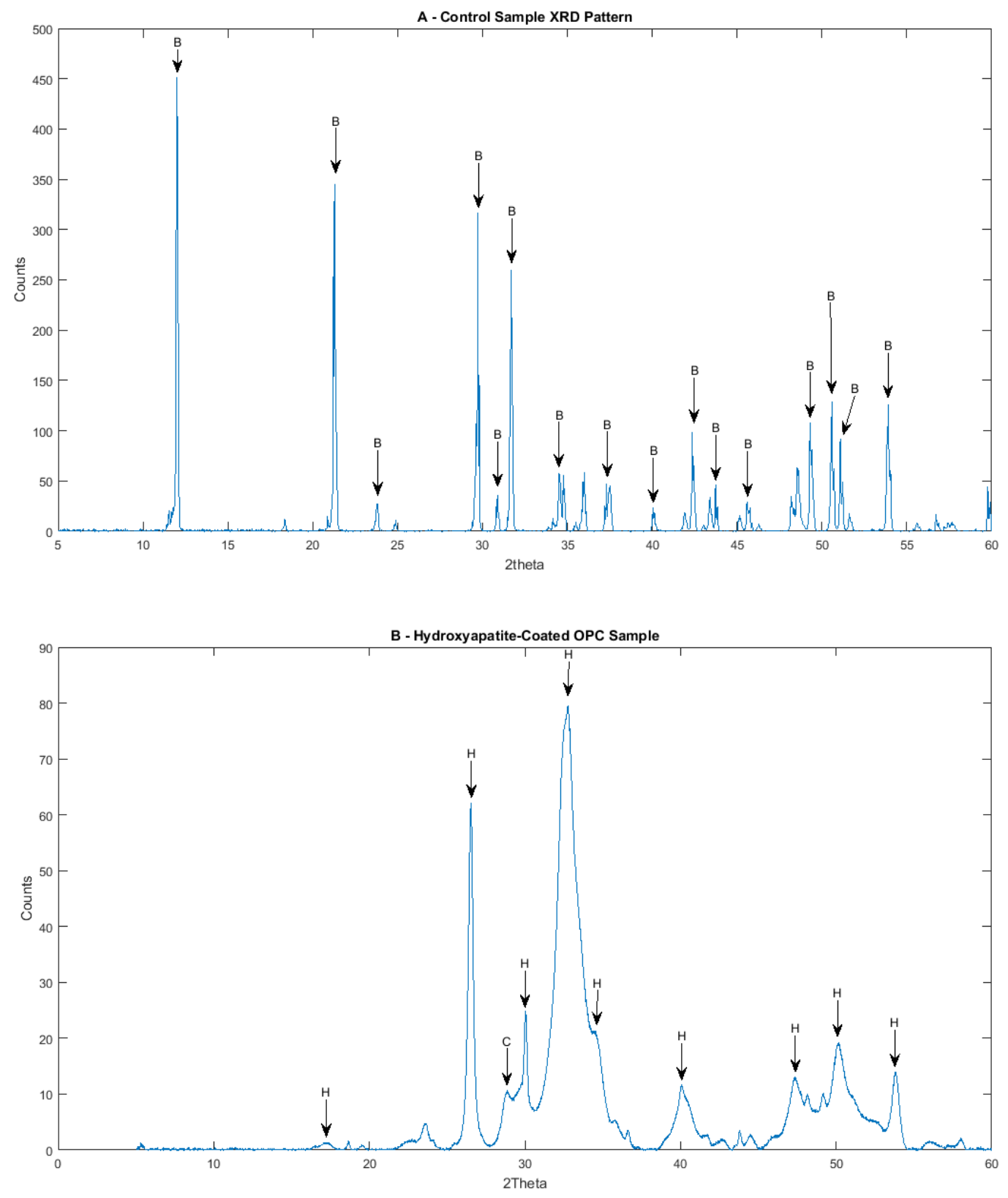

Figure 2: A) XRD pattern of control OPC sample. The pattern was matched to PDF 00-0090077 (Brushite) as annotated (B). B) XRD pattern of Pseudomonas-treated OPC sample. Reflections were matched to 289992-ICSD (hydroxyapatite) as annotated (H). A calcite peak (C) is also annotated on the test coupon, which was matched to 250-ICSD (PDF 01-0700095). The [002] reflection can be observed at c. $26^{\circ} 2$ theta. The size of the hydroxyapatite crystallites based on the [002] reflection FWHM was calculated as c. 30nm using Scherrer's equation. 

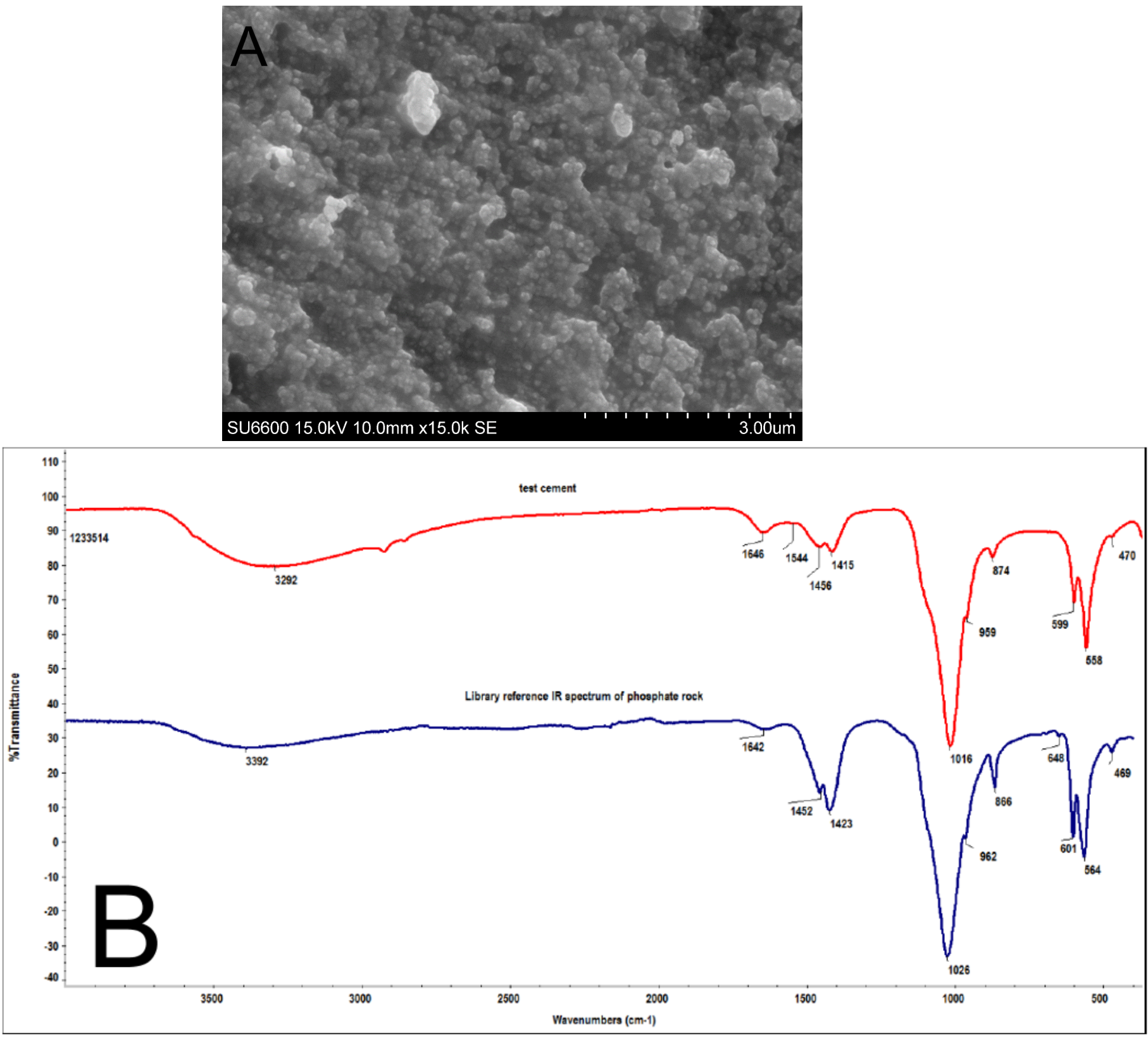

Figure 3: A) SEM micrograph of hydroxyapatite layer deposited onto the OPC substrate. Image is of a polished cross-section of the hydroxyapatite layer. The hydroxyapatite presents a relatively coarse morphology, consisting of an assembly of nanoscale particles. Individual particle sizes are $30-100 \mathrm{~nm}$. B) The IR spectrum of surface material from the same sample, which indicated that it consisted primarily of a phosphate, with a structure similar to hydroxyapatite (formula, $\mathrm{Ca}_{10}\left(\mathrm{PO}_{4}\right)_{6} \mathrm{OH}_{2}$ ) and some $\mathrm{CaCO}_{3}$. It showed strong similarities to a reference IR spectrum of a phosphate rock which contains hydroxyapatite 


\subsection{Interactions between Pseudomonas fluorescens, OPC, and Hy- droxyapatite}

Pseudomonasand other bacteria are capable of producing nano-hydroxyapatite with physical properties very similar to natural bone material. ${ }^{31}$ Research on the bacterial generation of hydroxyapatite has generally required immobilisation of the bacteria on a matrix, such as sol-gels or alginate beads, prior to mineral formation; ${ }^{31}$ and the addition of a specific calcium source to stimulate hydroxyapatite production. ${ }^{32}$

It is significant that these steps are not necessary when bio-hydroxyapatite is generated on an OPC substrate material, as shown in this study. OPC has several properties which may have contributed to this. The OPC block provides a fixed substrate for hydroxyapatite deposition; negating the requirement for a sol-gel or similar matrix. In previous studies, calcium phosphate loading was found to be necessary when generating bio-hydroxyapatite on polyurethane or titanium, ${ }^{31}$ and the addition of calcium and phosphorous donors was also necessary when using sol-gel or alginate substrates. ${ }^{32}$ In the case of OPC, calcium is present in sufficient quantities (Table 1) both on the OPC surface and in the surrounding solution.

$\mathrm{A} \mathrm{pH}$ of 7.4 or higher is necessary for the chemical synthesis of hydroxyapatite. ${ }^{23}$ For chemical deposition of biomimetic hydroxyapatite, $\mathrm{pH}$ values ranging from 7.3-11.5 are typical, ${ }^{19,22,33-35}$ and the presence of calcium and phosphate precursors is necessary. Previous studies of biogenic hydroxyapatite production involve multi-step processes and use a controlled rise in $\mathrm{pH}{ }^{31,32}$ In this work, a rise in $\mathrm{pH}$ was observed in both the control and test systems, compared to the initial pH of the LB broth (Table 2), despite the presence of the phosphate $\mathrm{pH}$ buffer. This rise in $\mathrm{pH}$ is due to $\mathrm{Ca}(\mathrm{OH})_{2}$ leaching from the cement. In the control system, the $\mathrm{pH}$ rise was limited to $\mathrm{pH} 7.4$ (from an initial $\mathrm{pH}$ of $\mathrm{pH} 6.2$ ); in the absence of higher $\mathrm{pH}$, brushite was formed rather than hydroxyapatite. However, in the systems containing $P$. fluorescens, the increase was much greater, rising to $\mathrm{pH} 9.1$. In the course of this work, we identified that attempting to grow $P$. fluorescens in this batch reaction system with no $\mathrm{pH}$ buffering capacity was not possible, due to an almost immediate 
rise in growth medium $\mathrm{pH}$ to 12.5 which inhibits $P$. fluorescens growth.

P. fluorescens is a non-lactose fermenting micro-organism. Consequently, in a growth medium such as LB broth which contains amino acids as the carbon and nitrogen sources, P. fluorescens will secrete ammonia, amines, and amides as by-products from the metabolic breakdown of amino acids. ${ }^{36}$ The secretion of these metabolic products accounts for the $\mathrm{pH}$ increase observed in the medium, which was greater than that caused by OPC alone (Table 2). The generation of an alkaline micro-environment around the bacterial cell is a well characterised process which occurs during the initial stages of calcite biomineralisation, with oxidative deamination of amino acids and the resulting increase in $\mathrm{pH}$ associated with calcium carbonate precipitation. ${ }^{37,38}$

The metabolic generation of ammonia by Pseudomonas can be expected to instigate a similar reaction to that which occurs during the generation of urolithiasis-associated struvite, calcite, and hydroxyapatite crystals by non-lactose fermenting micro-organisms such as Pseudomonas, Staphylococcus, Klebsiella, and Proteus. ${ }^{39-42}$ The reaction mechanism behind the bacterial production of struvite has also been linked to the generation of hydroxyapatite under conditions of increased calcium concentration such as the use of calcium acetate by Sanchez-Roman et al, (2007) ${ }^{39}$ but a cement substrate has never been used before in this context.

The reaction produces ammonium phosphates, via the bacterial generation of ammonia in a reaction system containing phosphates. ${ }^{43}$ Ammonium phosphates are used as chemical precursors to hydroxyapatite, and have been used as such for the consolidation of limestones. ${ }^{44-46}$ Hydroxyapatite does not form when OPC is placed in buffered LB broth without Pseudomonas (Fig 1B). We propose that in our system, P. fluorescens breaks down amino acids, and in doing so raises the $\mathrm{pH}$ and generates ammonia, leading to the formation of ammonium phosphate precursors for hydroxyapatite formation. We have identified that $P$. fluorescens generates ammonium phosphate minerals when grown in buffered LB medium (Fig 4), using a combination of optical microscopy and XRD analysis. It has been noted that 
hydroxyapatite production using carbonate and ammonium phosphate precursors occurs at room temperature and pressure, ${ }^{45}$ which is in agreement with the findings of this work; and supports this proposed chemical mechanism underlying hydroxyapatite production by Pseudomonas. The synergistic interactions between bacterial metabolism, the growth medium, and the OPC substrate which lead to hydroxyapatite biomineralisation are summarised in Figure 5 . 

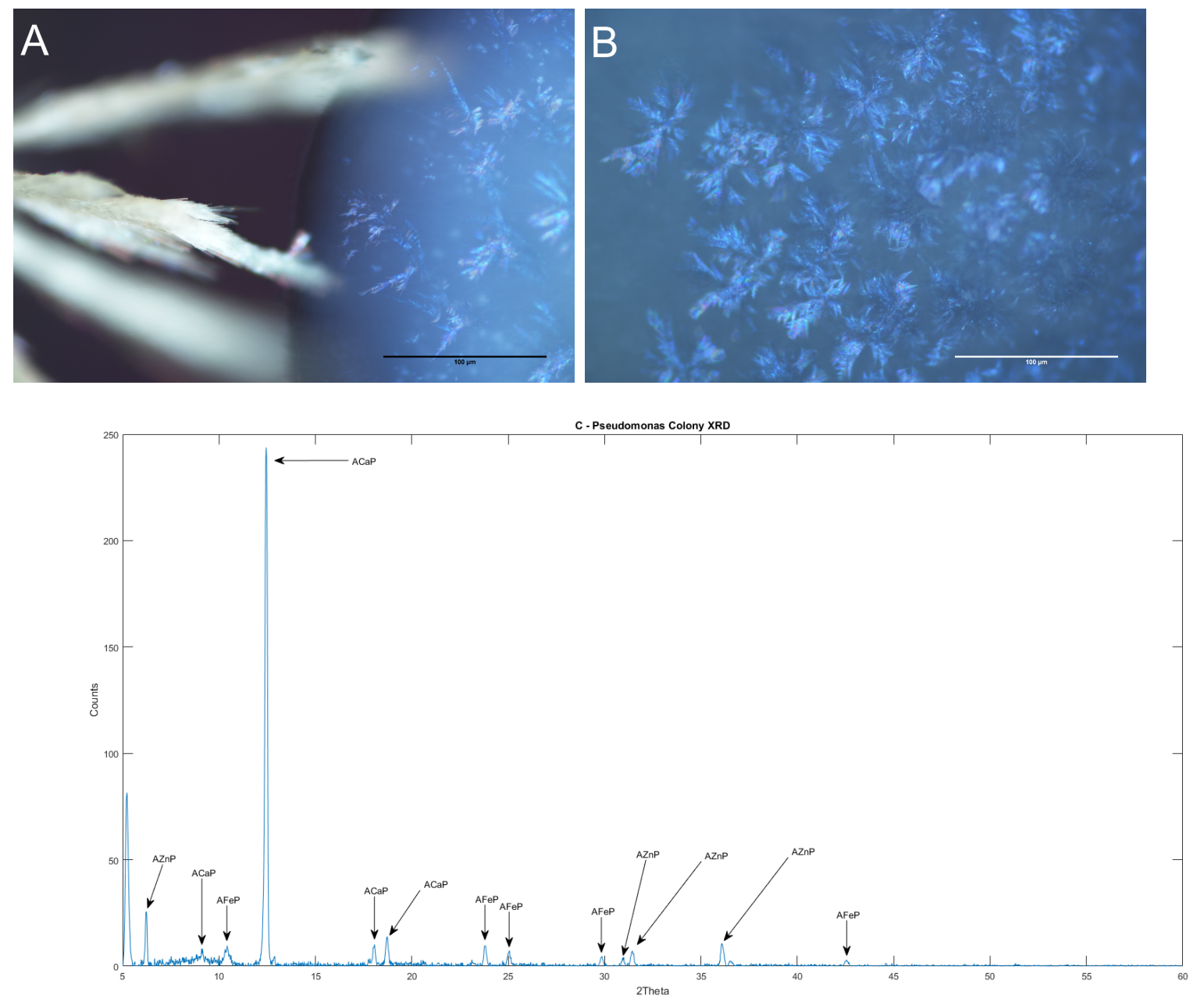

Figure 4: A) Cross-polarised transmitted light microscopy image of ammonium phosphate crystals precipitated into buffered LB agar by $P$. fluorescens. The image shows the edge of a single P. fluorescens colony (right hand side) on an agar plate. Precipitation can be observed both within the colony itself, and projecting from the surface of the colony as dendrites. B) Cross-polarised transmitted light microscopy image of ammonium phosphate crystals precipitated into buffered LB agar. The image shows the central portion of single $P$. fluorescens colony on an agar plate. Crystalline precipitates are observed to be dispersed as dendritic crystals throughout the bacterial colony. C) XRD patterns collected from extracted crystalline material after 12 days of incubation. Peaks annotated 'ACaP' were matched to PDF 00-035-0226 (ammonium calcium phosphate hydrate). Peaks annotated 'AFeP' were matched to PDF 00-031-0053 (ammonium iron phosphate hydrate). Peaks annotated 'AZnP' were matched to PDF 00-020-1429 (ammonium zinc phosphate hydrate).

It has been observed that bacteria control the process of bio-mineralisation, facilitating nucleation and growth of the mineral particles. ${ }^{47}$ This is perhaps best characterised in the bio-mineralisation of calcium carbonate, ${ }^{38,47,48}$ as well as magnetite deposition by the 
magnetotactic bacteria, and silica deposition by diatoms. ${ }^{49}$ The EPS skeleton generated by bacteria has been used as a nucleation point for the crystallisation of hydroxyapatite on titanium and foam substrates. ${ }^{32}$ In this context, the mechanism for this deposition process has been observed to use the cohesive, tightly adherent biofilm generated by the bacterium Serratia, which acts as a scaffold for the enzymatically mediated nucleation of calcium phosphate minerals. ${ }^{32}$ The biofilm matrix produces a confined 'reaction space' which prevents agglomeration of the nanocrystals, aiding the generation of nanoscale hydroxyapatite particles. ${ }^{32}$ We observed that $P$. fluorescens generates a biofilm scaffold (Fig 6) which may support the nucleation process.

In the built environment, hydroxyapatite coatings can be expected to reduce the dissolution of calcites in limestone and marble; ${ }^{45}$ however this protection has been observed to be less than anticipated. ${ }^{8}$ This has been partially attributed to the high thickness of the HAP films being prone to cracking, as well as layer porosity. ${ }^{8}$ Limestone is also a significant component of the OPC material (CEM-II/A-LL) used in this study, consisting of 6-20\% of the OPC mass. ${ }^{50} \mathrm{XRF}$ analysis of our cement finds a $\mathrm{CaO}$ weight $\%$ of $66 \%$ (Table 1), literature values of the $\mathrm{CaO}$ content of marbles and limestones are relatively close to this value, with measurements of approximately $55 \%$ appearing typical. ${ }^{51,52}$ As such, we would suggest that limestone and marble are likely to be suitable alternative substrate materials for the deposition process described in this work. 

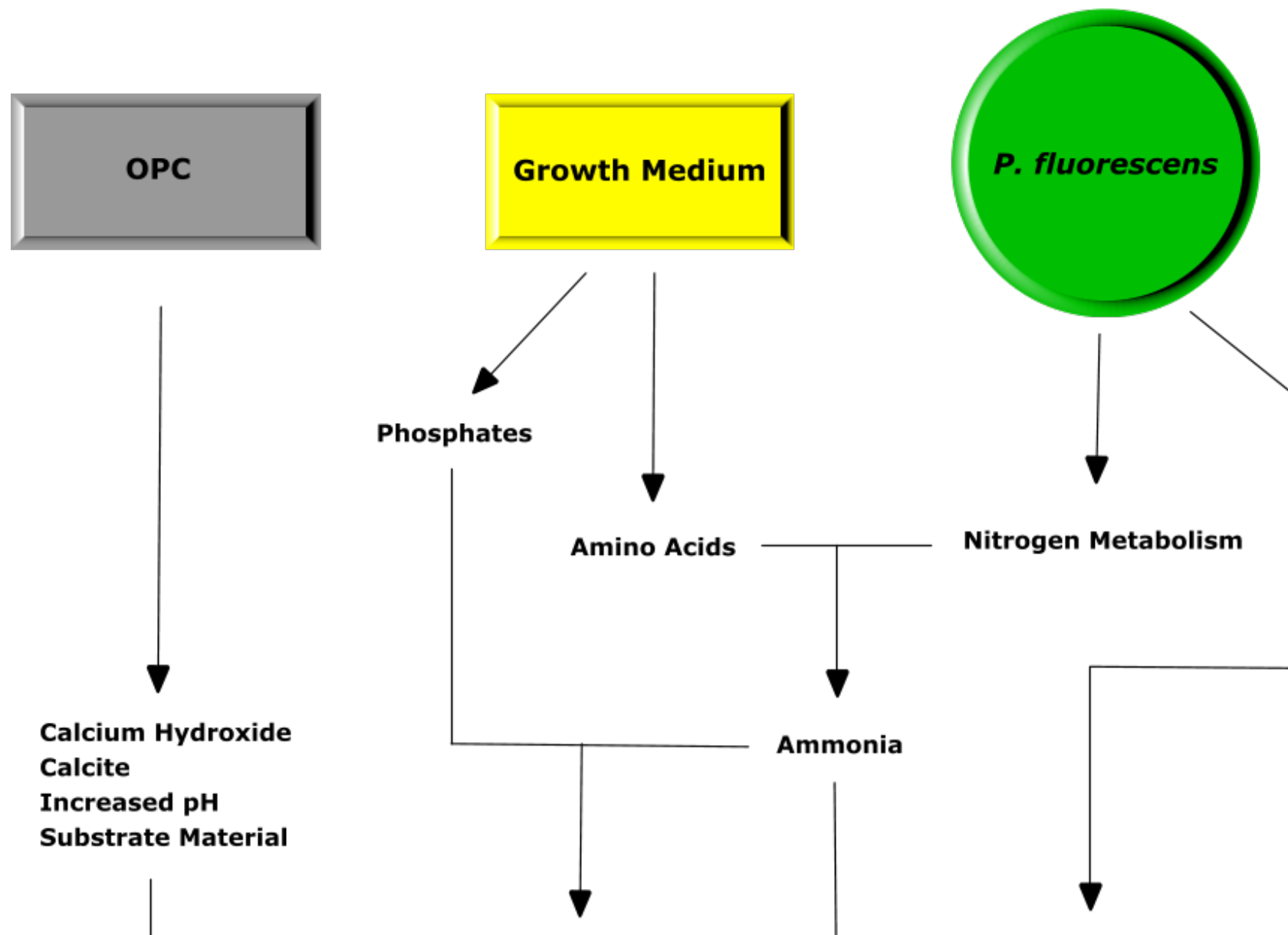

Nitrogen Metabolism

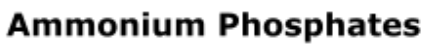

\section{Ammonia}
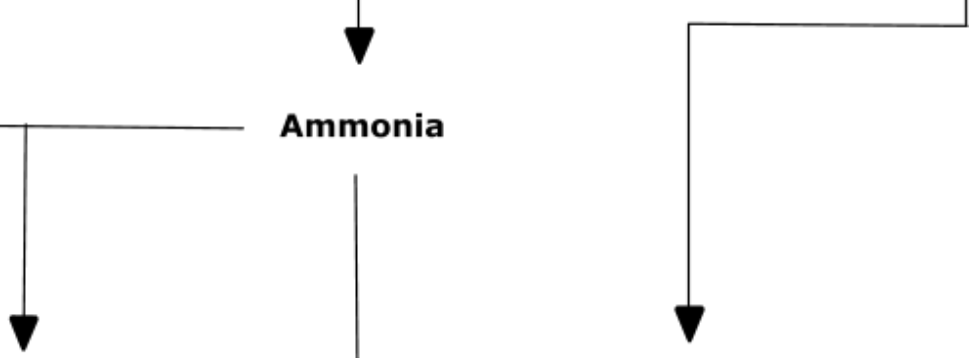

Biofilm Matrix Formation

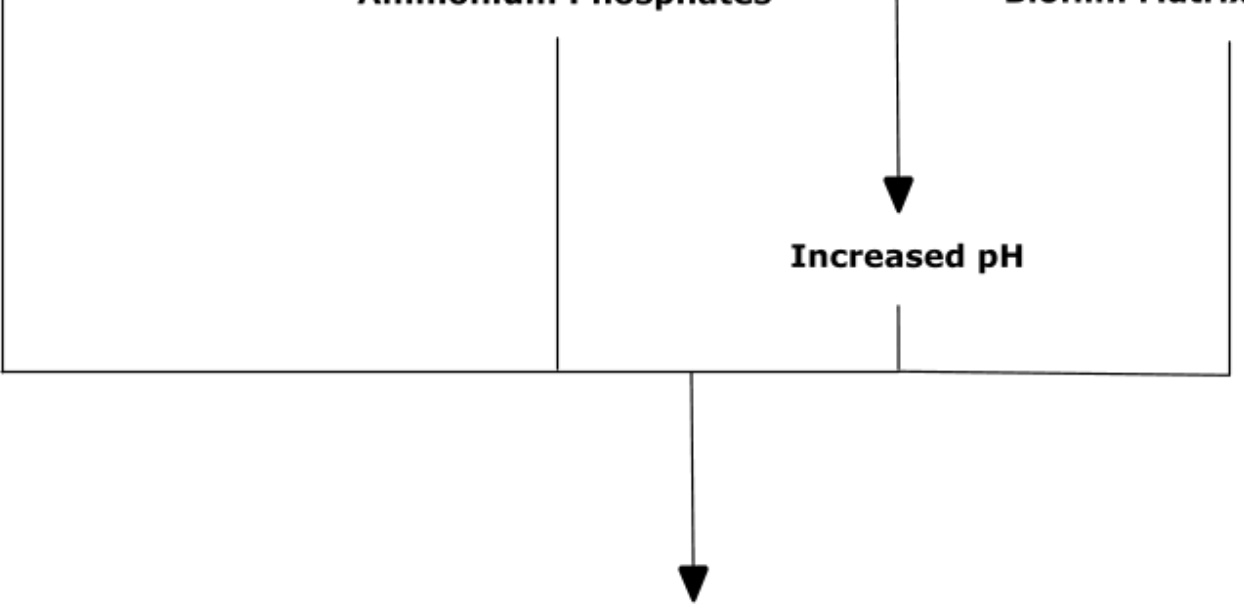

Hydroxyapatite Biomineralisation

Figure 5: A flow chart illustrating the synergistic interactions between components in the reaction system, which produces hydroxyapatite biomineralisation on the surface of the OPC substrate. 


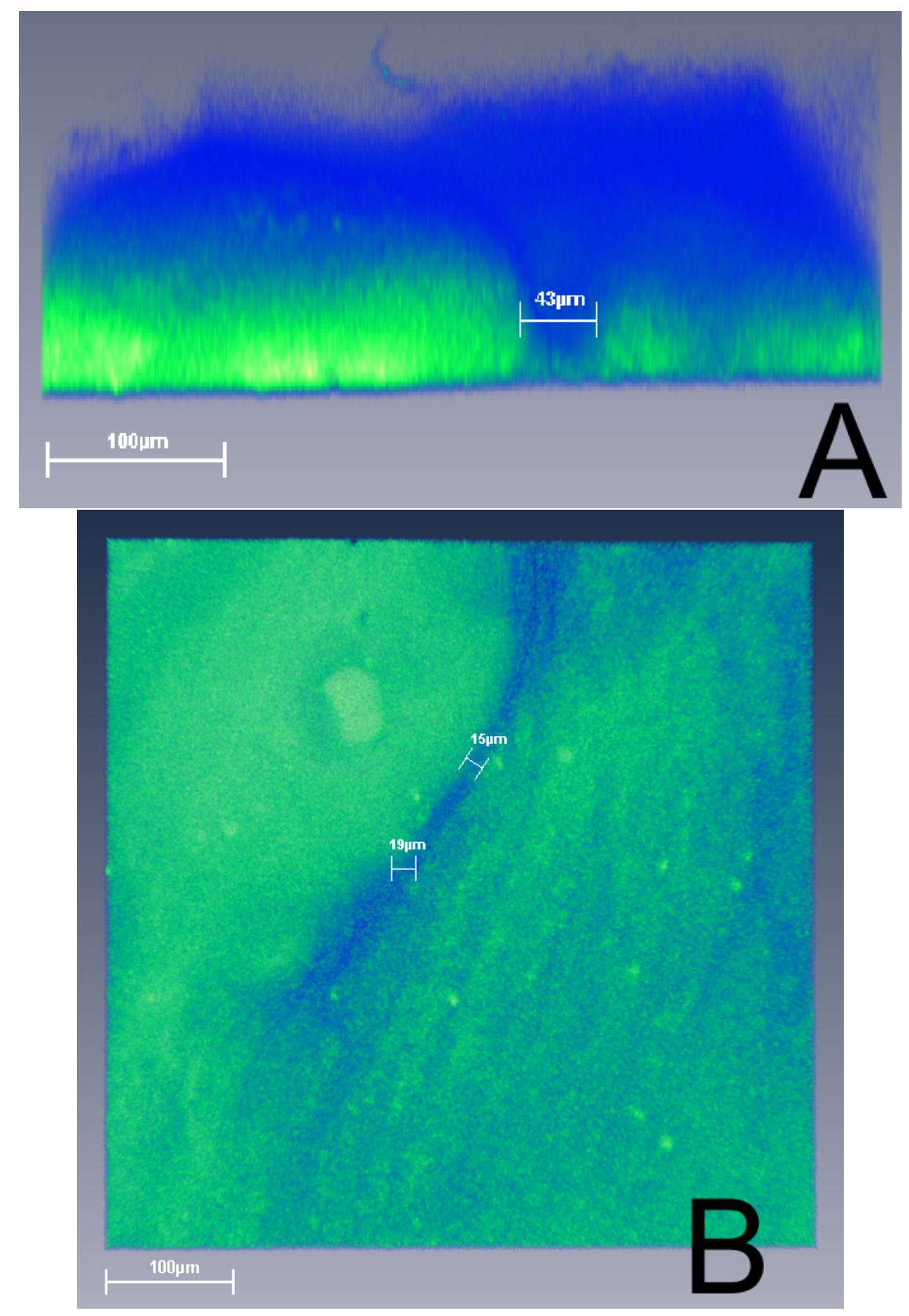

Figure 6: Confocal microscopy images of a P. fluorescens biofilm on the surface of an OPC coupon. The image shows A) XZ and B) XY projections of the biofilm. A 'channel' of lower biofilm density can be observed, measuring approximately $43 \mu \mathrm{m}$ (annotated) across at the 'entry' point as observed on the XZ projection and narrowing to breadths of approximately $15-19 \mu \mathrm{m}$ (annotated) further into the 'channel' as observed in the XY projection. Blue represents areas of lower biofilm signal intensity, green represents areas of higher biofilm signal intensity; the highest signal intensity areas are shown in Yellow. 


\section{Tables}

\subsection{XRF Table}

Table 1: XRF Analysis

\begin{tabular}{llll}
\hline Analyte & Chemical Form & Dry OPC Powder & Hydrated OPC Paste $^{1}$ \\
\hline Calcium & $\mathrm{CaO}$ & 72.05 & 66.15 \\
Silica & $\mathrm{SiO}_{2}$ & 21.76 & 21.08 \\
Alumina & $\mathrm{Al}_{2} \mathrm{O}_{3}$ & 5.1 & 4.67 \\
Iron & $\mathrm{Fe}_{2} \mathrm{O}_{3}$ & 3.34 & 3.17 \\
Magnesium & $\mathrm{MgO}$ & 2.17 & 1.72 \\
Titanium & $\mathrm{TiO}_{2}$ & 0.21 & 0.2 \\
Sodium & $\mathrm{Na}_{2} \mathrm{O}$ & 0.33 & 0.09 \\
Potassium & $\mathrm{K}_{2} \mathrm{O}$ & 0.87 & 0.13 \\
Phosphorus & $\mathrm{P}_{2} \mathrm{O}_{5}$ & 0.11 & 0.09 \\
Zinc & $\mathrm{ZnO}_{\text {Sulfur Trioxide }} \mathrm{SO}_{3}$ & 0.12 & 0.12 \\
Sulfur & $\mathrm{S}$ & 3.38 & 2.29 \\
& \multicolumn{3}{l}{ All values are weight\% } \\
\hline
\end{tabular}

\section{$4.2 \mathrm{pH}$ Table}

Table 2: pH Measurements

\begin{tabular}{cc}
\hline Sample & $\mathrm{pH}^{2}$ \\
\hline Test & $9.065 \pm 0.027$ \\
Control & $7.445 \pm 0.201$ \\
Buffered LB (No OPC) & $6.204 \pm 0.007$ \\
\cline { 2 - 2 } . & All values are mean of $\mathrm{n}=3 \pm$ standard error of the mean
\end{tabular}

\section{Conclusions}

We have demonstrated for the first time that a biogenic hydroxyapatite can be directly biomineralised onto the surface of Ordinary Portland Cement, and described the biochemical mechanisms behind this deposition process. Both XRD and FT-IR analysis illustrate that the disorder, carbonation, crystal structure, and crystallite size of this deposited hydroxyapatite are similar to natural apatite materials; with associated advantages over synthetic apatites 
which have been described elsewhere. ${ }^{13,16-18}$ The direct biomineralisation of hydroxyapatite on OPC has never been reported before; and as such the biochemical mechanisms underlying this unique synergistic process have not been investigated or described in the literature to date.

The described method presents a rapid, simple, and cheap method of promoting in-situ biomineralisation of hydroxyapatite onto the OPC substrate, with associated advantages arising from the formation of this biogenic hydroxyapatite in comparison with more widely studied biomimetic hydroxyapatites. Significantly, we have observed that the bacterium Pseudomonas fluorescens will produce this biogenic hydroxyapatite under mild reaction conditions within a relatively simple reaction system, which uses the chemical and physical properties of a Portland cement substrate material. We suggest that OPC has properties which make it a favourable substrate for bacterial hydroxyapatite deposition, namely the presence of accessible calcium, ${ }^{39}$ which in combination with the $\mathrm{pH}$ rise and ammonia production arising from microbial metabolic processes, favours hydroxyapatite formation. ${ }^{23}$ There is significant potential to investigate this deposition process on other calcium-rich substrate materials, such as marbles and limestones.

\section{Acknowledgement}

The authors gratefully acknowledge the Engineering and Physical Sciences Research Council (EPSRC) for funding the research as a Doctoral Training Partnership awared to Andrea Hamilton, Joanna C Renshaw, and Ronald J Turner. Thanks to Gerry Johnston, Fiona Sillars, and Tiziana Marrocco of the University of Strathclyde AMRL for assistance with SEM-EDS and XRD data collection; and thanks to Gillian Robb and Graeme MacKenzie of the University of Strathclyde Centre for Biophotonics for assistance with confocal microscopy. 


\section{References}

(1) Elliott, J. C. Structure and Chemistry of the Apatites and Other Calcium Orthophosphates; Elsevier Science, 1994; pp 1-389.

(2) Lin, K.; Xia, L.; Gan, J.; Zhang, Z.; Chen, H.; Jiang, X.; Chang, J. Tailoring the nanostructured surfaces of hydroxyapatite bioceramics to promote protein adsorption, osteoblast growth, and osteogenic differentiation. ACS Appl. Mater. Interfaces 2013, $5,8008-8017$.

(3) Mistry, S.; Kundu, D.; Datta, S.; Basu, D. Comparison of bioactive glass coated and hydroxyapatite coated titanium dental implants in the human jaw bone. Aust. Dent. J. 2011, 56, 68-75.

(4) Sarath Chandra, V.; Baskar, G.; Suganthi, R. V.; Elayaraja, K.; Ahymah Joshy, M. I.; Sofi Beaula, W.; Mythili, R.; Venkatraman, G.; Narayana Kalkura, S. Blood compatibility of iron-doped nanosize hydroxyapatite and its drug release. ACS Appl. Mater. Interfaces 2012, 4, 1200-1210.

(5) Moursi, A. M.; Winnard, A. V.; Winnard, P. L.; Lannutti, J. J.; Seghi, R. R. Enhanced osteoblast response to a polymethylmethacrylate-hydroxyapatite composite. Biomaterials 2002, 23, 133-144.

(6) Dalby, M. J.; Di Silvio, L.; Harper, E. J.; Bonfield, W. Increasing hydroxyapatite incorporation into poly(methylmethacrylate) cement increases osteoblast adhesion and response. Biomaterials 2002, 23, 569-576.

(7) Dalby, M. J.; Di Silvio, L.; Harper, E. J.; Bonfield, W. Initial interaction of osteoblasts with the surface of a hydroxyapatite-poly(methylmethacrylate) cement. Biomaterials 2001, 22, 1739-1747. 
(8) Graziani, G.; Sassoni, E.; Franzoni, E.; Scherer, G. W. Hydroxyapatite coatings for marble protection: Optimization of calcite covering and acid resistance. Appl. Surf. Sci. 2016, 368, 241-257.

(9) Sassoni, E.; Graziani, G.; Franzoni, E. An innovative phosphate-based consolidant for limestone. Part 2: Durability in comparison with ethyl silicate. Constr. Build. Mater. 2016, 102, 931-942.

(10) Franzoni, E.; Sassoni, E.; Graziani, G. Brushing, poultice or immersion? The role of the application technique on the performance of a novel hydroxyapatite-based consolidating treatment for limestone. Journal of Cultural Heritage 2015, 16, 173-184.

(11) Yang, F.; Liu, Y. Artificial hydroxyapatite film for the conservation of outdoor marble artworks. Mater. Lett. 2014, 124, 201-203.

(12) Sassoni, E.; Graziani, G.; Franzoni, E. An innovative phosphate-based consolidant for limestone. Part 1: Effectiveness and compatibility in comparison with ethyl silicate. Constr. Build. Mater. 2016, 102, 918-930.

(13) Handley-Sidhu, S.; Renshaw, J. C.; Moriyama, S.; Stolpe, B.; Mennan, C.; Bagheriasl, S.; Yong, P.; Stamboulis, A.; Paterson-Beedle, M.; Sasaki, K.; Pattrick, R. A. D.; Lead, J. R.; MacAskie, L. E. Uptake of Sr $2+$ and Co $2+$ into biogenic hydroxyapatite: Implications for biomineral ion exchange synthesis. Environ. Sci. Technol. 2011, 45, 6985-6990.

(14) Sasaki, K.; Goto, T. Immobilization of $\mathrm{Sr} 2+$ on naturally derived hydroxyapatite by calcination of different species of fish bones and influence of calcination on ion-exchange efficiency. Ceram. Int. 2014, 40, 11649-11656.

(15) Chen, X.; Wright, J. V.; Conca, J. L.; Peurrung, L. M. Evaluation of Heavy Metal Remediation Using Mineral Apatite. Water, Air, Soil Pollut. 2000, 98, 57-78. 
(16) Li, L.; Pan, H.; Tao, J.; Xu, X.; Mao, C.; Gu, X.; Tang, R. Repair of enamel by using hydroxyapatite nanoparticles as the building blocks. J. Mater. Chem. 2008, 18, 4079.

(17) Sadat-Shojai, M.; Khorasani, M. T.; Dinpanah-Khoshdargi, E.; Jamshidi, A. Synthesis methods for nanosized hydroxyapatite with diverse structures. Acta Biomater. 2013, 9, 7591-7621.

(18) Pinchuk, N.; Parkhomey, O.; Sych, O. In Vitro Investigation of Bioactive GlassCeramic Composites Based on Biogenic Hydroxyapatite or Synthetic Calcium Phosphates. Nanoscale Res. Lett. 2017, 12, 111.

(19) Rusu, V. M.; Ng, C. H.; Wilke, M.; Tiersch, B.; Fratzl, P.; Peter, M. G. Size-controlled hydroxyapatite nanoparticles as self-organized organic-inorganic composite materials. Biomaterials 2005, 26, 5414-5426.

(20) Liu, Q.; Huang, S.; Matinlinna, J. P.; Chen, Z.; Pan, H. Insight into biological apatite: physiochemical properties and preparation approaches. BioMed Res. Int. 2013, 2013.

(21) Abràmoff, M. D.; Magalhães, P. J.; Ram, S. J. Image processing with imageJ. Biophotonics International 2004, 11, 36-41.

(22) Müller, L.; Conforto, E.; Caillard, D.; Müller, F. A. Biomimetic apatite coatingsCarbonate substitution and preferred growth orientation. Biomol. Eng. 2007, 24, 462466.

(23) Eiden-Aßmann, S.; Viertelhaus, M.; Heiß, A.; Hoetzer, K. A.; Felsche, J. The influence of amino acids on the biomineralization of hydroxyapatite in gelatin. J. Inorg. Biochem. 2002, 91, 481-486.

(24) Kalita, S. J.; Bhardwaj, A.; Bhatt, H. A. Nanocrystalline calcium phosphate ceramics in biomedical engineering. Mater. Sci. Eng., C 2007, 27, 441-449.

(25) Tanner, K. Small but extremely tough. Science 2012, 336, 1237-1238. 
(26) Beniash, E. Biominerals- hierarchical nanocomposites : the example of bone. Wiley Interdiscip. Rev.: Nanomed. Nanobiotechnol. 2011, 3, 47-69.

(27) Fleet, M. E. Carbonated Hydroxyapatite: Materials, Synthesis, and Applications; Pan Stanford Publishing, 2014.

(28) Ulian, G.; Valdrè, G.; Corno, M.; Ugliengo, P. Periodic ab initio bulk investigation of hydroxylapatite and type a carbonated apatite with both pseudopotential and allelectron basis sets for calcium atoms. Am. Mineral. 2013, 98, 410-416.

(29) Huang, S. B.; Gao, S. S.; Yu, H. Y. Effect of nano-hydroxyapatite concentration on remineralization of initial enamel lesion in vitro. Biomed. Mater. 2009, 4, 1-6.

(30) da Cunha, M. R.; Menezes, F. A.; dos Santos, G. R.; Pinto, C. A. L.; Barraviera, B.; Martins, V. d. C. A.; Plepis, A. M. d. G.; Ferreira Junior, R. S. Hydroxyapatite and a New Fibrin Sealant Derived from Snake Venom as Scaffold to Treatment of Cranial Defects in Rats. Mater. Res. (Sao Carlos, Braz.) 2015, 18, 196-203.

(31) Khanafari, A.; Akbari, T.; Sohrabi, M. R. Comparison of nano-hydroxyapatite productivity by Pseudomonas aeruginosa and Serratia marcescense through encapsulation method Nano-hydroxyapatite productivity by encapsulation method. Nanomed. J. 2014, 1, 276-284.

(32) Macaskie, L. E.; Yong, P.; Paterson-Beedle, M.; Thackray, A. C.; Marquis, P. M.; Sammons, R. L.; Nott, K. P.; Hall, L. D. A novel non line-of-sight method for coating hydroxyapatite onto the surfaces of support materials by biomineralization. J. Biotechnol. 2005, 118, 187-200.

(33) Roveri, N.; Battistella, E.; Bianchi, C. L.; Foltran, I.; Foresti, E.; Iafisco, M.; Lelli, M.; Naldoni, A.; Palazzo, B.; Rimondini, L. Surface enamel remineralization: biomimetic apatite nanocrystals and fluoride ions different effects. J. Nanomater. 2009, 2009, 8. 
(34) Li, S.; Wu, H. H.; Xu, G. J.; Xiao, X. F. Facile Biomimetic Fabrication of Hollow Hydroxyapatite with Hierarchically Porous Microstructure Using Hyperbranched Gemini Surfactant as Template. Adv. Mater. Res. (Durnten-Zurich, Switz.) 2014, 1015, $355-358$.

(35) Zhang, Q.; Leng, Y. Electrochemical activation of titanium for biomimetic coating of calcium phosphate. Biomaterials 2005, 26, 3853-3859.

(36) Rhodes, M. E. The Characterization of Pseudomonas fluorescens. J. Gen. Microbiol. 1959, 21, 221-263.

(37) Dupraz, S.; Parmentier, M.; Ménez, B.; Guyot, F. Experimental and numerical modeling of bacterially induced $\mathrm{pH}$ increase and calcite precipitation in saline aquifers. Chem. Geol. 2009, 265, 44-53.

(38) Rodriguez-Navarro, C.; Jroundi, F.; Schiro, M.; Ruiz-Agudo, E.; GonzálezMuñoz, M. T. Influence of substrate mineralogy on bacterial mineralization of calcium carbonate: Implications for stone conservation. Appl. Environ. Microbiol. 2012, 78, 4017-4029.

(39) Sánchez-Román, M.; Rivadeneyra, M. A.; Vasconcelos, C.; McKenzie, J. A. Biomineralization of carbonate and phosphate by moderately halophilic bacteria. FEMS Microbiol. Ecol. 2007, 61, 273-284.

(40) Grases, F.; Söhnel, O.; Vilacampa, A. I.; March, J. G. Phosphates precipitating from artificial urine and fine structure of phosphate renal calculi. Clin. Chim. Acta 1996, $244,45-67$.

(41) Kim, J. W.; Yoon, C. Y.; Park, H. S.; Lee, J. G.; Moon, D. G.; Oh, M. M. Precipitation of amorphous magnesium ammonium phosphate: Is it a precursor for staghorn stones? Urolithiasis 2014, 42, 283-284. 
(42) Palma, D.; Langston, C.; Gisselman, K.; McCue, J. Canine struvite urolithiasis. Compendium Contin. Educ. Vet. 2013, 35, 457-458.

(43) Beavon, J.; Heatley, N. G. The occurrence of struvite (magnesium ammonium phosphate hexahydrate) in microbial cultures. J. Gen. Microbiol. 1963, 31, 167-9.

(44) Sassoni, E.; Graziani, G.; Franzoni, E. Repair of sugaring marble by ammonium phosphate: Comparison with ethyl silicate and ammonium oxalate and pilot application to historic artifact. Mater. Des. 2015, 88, 1145-1157.

(45) Naidu, S. Novel Hydroxyapatite Coatings for the Conservation of Marble and Limestone. Ph.D. thesis, Princeton, 2014.

(46) Sassoni, E.; Naidu, S.; Scherer, G. W. The use of hydroxyapatite as a new inorganic consolidant for damaged carbonate stones. Journal of Cultural Heritage 2011, 12, 346355.

(47) Barabesi, C.; Galizzi, A.; Mastromei, G.; Rossi, M.; Tamburini, E.; Perito, B. Bacillus subtilis gene cluster involved in calcium carbonate biomineralization. J. Bacteriol. 2007, 189, 228-235.

(48) Zamarreño, D. V.; Inkpen, R.; May, E. Carbonate crystals precipitated by freshwater bacteria and their use as a limestone consolidant. Appl. Environ. Microbiol. 2009, 75, $5981-5990$.

(49) Bauerlein, E. Biomineralization of unicellular organisms: An unusual membrane biochemistry for the production of inorganic nano- and microstructures. Angew. Chem., Int. Ed. 2003, 42, 614-641.

(50) British Standards Institute, BS EN 197-1:2011 - Cement Part 1: Composition, Specifications and Conformity Criteria for Common Cements; 2011. 
(51) Vaggelli, G.; Serra, M.; Cossio, R.; Borghi, A. A New Approach for Provenance Studies of Archaeological Finds: Inferences from Trace Elements in Carbonate Minerals of Alpine White Marbles by a Bench-to-Top $\mu$-XRF Spectrometer. Int. J. Mineral. 2014, 2014 .

(52) Hemeda, S. Geotechnical Characterization of Sakakini's Palace Stones and Other Construction Materials, Cairo-Egypt. Geomaterials 2013, 3, 38-46. 


\section{Graphical TOC Entry}

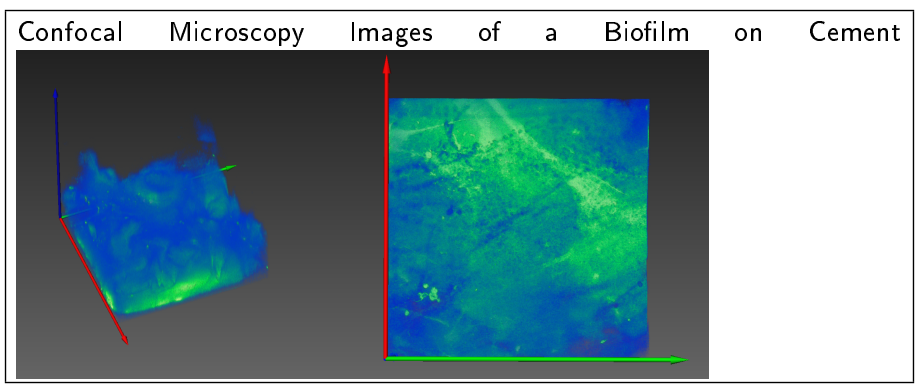

Some journals require a graphical entry for the Table of Contents. This should be laid out "print ready" so that the sizing of the text is correct. Inside the tocentry environment, the font used is Helvetica $8 \mathrm{pt}$, as required by Journal of the American Chemical Society.

The surrounding frame is $9 \mathrm{~cm}$ by $3.5 \mathrm{~cm}$, which is the maximum permitted for Journal of the American Chemical Society graphical table of content entries. The box will not resize if the content is too big: instead it will overflow the edge of the box.

This box and the associated title will always be printed on a separate page at the end of the document. 